\title{
The impact of histopathology and NAB2-STAT6 fusion subtype in classification and grading of meningeal solitary fibrous tumor/ hemangiopericytoma
}

\author{
Karen Fritchie ${ }^{1}$ (D) Kassandra Jensch ${ }^{2}$ - Evgeny A. Moskalev ${ }^{2}$. Alissa Caron ${ }^{1} \cdot$ Sarah Jenkins $^{3} \cdot$ Michael Link $^{4}$. \\ Paul D. Brown ${ }^{5}$. Fausto J. Rodriguez ${ }^{6}$. Andrew Guajardo ${ }^{6}$. Daniel Brat ${ }^{7}$. José E. Velázquez Vega ${ }^{8}$. Arie Perry ${ }^{9}$. \\ Ashley $\mathrm{Wu}^{9}$. David R. Raleigh ${ }^{10,11}$. Sandro Santagata ${ }^{12}$. David N. Louis ${ }^{13}$. Priscilla K. Brastianos ${ }^{14}$. \\ Alexander Kaplan ${ }^{15}$. Brian M. Alexander ${ }^{16} \cdot$ Sabrina Rossi $^{17} \cdot$ Fabio Ferrarese $^{18} \cdot$ Florian Haller $^{2} \cdot$ Caterina Giannini $^{1}$
}

Received: 15 October 2018 / Revised: 4 December 2018 / Accepted: 17 December 2018 / Published online: 24 December 2018

(c) The Author(s) 2018

\begin{abstract}
Meningeal solitary fibrous tumor (SFT)/hemangiopericytoma (HPC) is a rare tumor with propensity for recurrence and metastasis. Although multiple classification schemes have been proposed, optimal risk stratification remains unclear, and the prognostic impact of fusion status is uncertain. We compared the 2016 WHO CNS tumor grading scheme (CNS-G), a three-tier system based on histopathologic phenotype and mitotic count, to the 2013 WHO soft-tissue counterpart (ST-G), a two-tier system based on mitotic count alone, in a cohort of 133 patients [59 female, 74 male; mean age 54 years (range 20-87)] with meningeal SFT/HPC. Tumors were pathologically confirmed through review of the first tumor resection $(n=97)$, local recurrence $(n=35)$, or distant metastasis $(n=1)$. A STAT6 immunostain showed nuclear expression in 132 cases. NAB2-STAT6 fusion was detected in 99 of 111 successfully tested tumors (89\%) including the single STAT6 immunonegative tumor. Tumors were classified by CNS-G as grade $1(n=43), 2(n=41)$, or $3(n=49)$, and by ST-G as SFT $(n=84)$ or malignant SFT $(n=49)$. Necrosis was present in 16 cases $(12 \%)$. On follow-up, 42 patients had at least one subsequent recurrence or metastasis ( 7 metastasis only, 33 recurrence only, 2 patients had both). Twenty-nine patients died. On univariate analysis, necrosis $(p=0.002)$, CNS-G $(p=0.01)$, and ST-G $(p=0.004)$ were associated with recurrence-free (RFS) but not overall survival (OS). NAB2-STAT6 fusion type was not significantly associated with RFS or OS, but was associated with phenotype. A modified ST-G incorporating necrosis showed higher correlation with RFS $(p=0.0006)$ and remained significant $(p=0.02)$ when considering only the primary tumors. From our data, mitotic rate and necrosis appear to stratify this family of tumors most accurately and could be incorporated in a future grading scheme.
\end{abstract}

Keywords Meningeal hemangiopericytoma $\cdot$ Meningeal solitary fibrous tumor $\cdot$ NAB2-STAT6 $\cdot$ STAT6

Florian Haller and Caterina Giannini are co-senior authors.

Electronic supplementary material The online version of this article (https://doi.org/10.1007/s00401-018-1952-6) contains supplementary material, which is available to authorized users.

Karen Fritchie

Fritchie.Karen@Mayo.edu

Extended author information available on the last page of the article

\section{Introduction}

Meningeal solitary fibrous tumor (SFT)/hemangiopericytoma (HPC) is an often aggressive mesenchymal tumor of fibroblastic origin that arises from the cranial or spinal dura [14]. Although SFT and HPC were initially thought to represent distinct entities, the identification of NAB2-STAT6 fusion as a defining molecular alteration in both tumors has led to the unification of these entities at both dural and extra-dural sites. While the NAB2-STAT6 fusion seems to be unique to SFT/HPC, its detection may be difficult unless whole-genome sequencing is applied to detect breakpoints that occur both in exon and intron boundaries [5, 16]. STAT6 nuclear expression is accepted as a sensitive surrogate of 
all fusions, which causes consistent nuclear relocation of STAT6 $[9,14,17]$. Tumors arising from the meninges tend to exhibit high rates of local recurrence with propensity for metastasis outside the central nervous system (CNS), but prognostication on histopathology alone has been notoriously difficult regardless of site of origin. The current 2016 WHO CNS grading scheme incorporates phenotype and mitotic rate to stratify tumors into three groups (grade 1,2, and 3), while non-meningeal soft-tissue tumors are classified by mitotic rate alone into SFT and malignant SFT according to the 2013 WHO classification for soft-tissue tumors [10, 14]. Our earlier work suggested that tumors harboring the NAB2 exon 4-STAT6 exon 3 fusion variant exhibited morphologic features similar to the conventional solitary fibrous tumor, while there was a trend toward an association with the hemangiopericytoma phenotype and more aggressive behavior in tumors lacking this variant [11]. We studied a large series of patients with SFT/HPC from six tertiary care centers, to determine the best grading scheme for meningeal-based SFT/HPT and characterize the relationship of NAB2-STAT6 fusion status with phenotype and prognosis.

\section{Materials and methods}

This study was reviewed and approved by the Institutional Review Board at all participating institutions.

\section{Case selection}

A cohort of 133 patients with meningeal SFT/HPC (74 males; 59 females) was identified from six tertiary care centers. They ranged in age from 17 to 78 years (median 49.2) at the initial diagnosis. Tumors were pathologically confirmed through review of the first tumor resection $(n=97)$, local recurrence $(n=35)$, or distant metastasis $(n=1)$. Data regarding treatment and clinical follow-up were obtained at the respective institutions based on review of the clinical records.

\section{Histologic review}

Archived H\&E-stained sections were classified phenotypically as 'SFT', 'HPC', or tumors with intermediate morphological features between HPC and SFT (Fig. 1). SFTs were low-to-moderate cellular tumors composed of spindled to ovoid-shaped cells arranged around branching blood vessels with variable stromal and perivascular hyalinization. HPCs harbored ovoid-to-round cells, often in a sheet-like growth pattern, with high $\mathrm{N}: \mathrm{C}$ ratios and scant, amphophilic to-clear cytoplasm. Although branching blood vessels were typically present, the vasculature of HPC was frequently less conspicuous than the conventional SFT, and stromal hyalinization was generally absent. Tumors showing the features of both SFT and HPC were considered intermediate. Mitotic rate was assessed by scanning all available $\mathrm{H} \& \mathrm{E}$ slides and counting mitoses $($ at $\times 400)$ in ten consecutive fields in the areas of highest mitotic activity. The presence of necrosis was recorded for all tumors.

\section{Classification and grading}

Tumors were classified and graded according to the most recent CNS (2016) (CNS-G) and Soft Tissue (2013) (STG) WHO classification schemes (Fig. 2). According to the CNS-G, tumors with a classic SFT histopathological phenotype and fewer than five mitoses $(\times 10 \mathrm{HPF})$ were considered grade 1; tumors with intermediate or HPC phenotype and fewer than five mitoses $(\times 10 \mathrm{HPF})$ were considered grade 2 ; tumors with five or more mitoses $(\times 10 \mathrm{HPF})$ were considered grade 3 irrespective of their histopathological phenotype. According to the ST-G, irrespective of their histopathological phenotype, tumors with fewer than five mitoses $(\times 10 \mathrm{HPF})$ were considered SFT and tumors with five or more mitoses $(\times 10 \mathrm{HPF})$ were considered malignant SFT.

\section{Immunohistochemistry}

A representative tissue block from each tumor was selected and stained for STAT6 (Santa Cruz Biotechnology, CA, mouse monoclonal antibody, 1:50) and CD34 (Novocastra, liquid mouse monoclonal antibody, 1:100). CD34 immunoreactivity was recorded as negative $(<5 \%)$, focal $(5-50 \%)$, or diffuse ( $>50 \%)$, while nuclear expression of STAT6 was scored as negative (when nuclear STAT6 expression was not present in tumor cells) or positive (when the cells showed definite STAT6 nuclear expression at least focally in the tumor).

\section{Molecular studies}

RNA extraction was performed by the Mayo Clinic Pathology Research Core using the Qiagen miRNeasy FFPE kit by methods previously described by Wang et al. [20]. RT-PCR of hypoxanthine phosphoribosyltransferase 1 (HPRT1) was used as control for RNA integrity as described previously $[3,11]$ and samples with degraded RNA were discarded to avoid generation of false-negative cases. In a first step, we screened for the presence of the most common NAB2-STAT6 fusions exon 4-exon 2, exon 4-exon 3, exon 6-exon 16, and exon 6-exon 17 using the well-established single-plex PCR with subsequent agarose gel electrophoresis, identifying NAB2-STAT6 gene fusions in 52 samples. In a next step, samples with good RNA quality but no result in the single-plex PCRs were analyzed by next-generation sequencing using the Archer FusionPlex Sarcoma Kit (ArcherDx, 

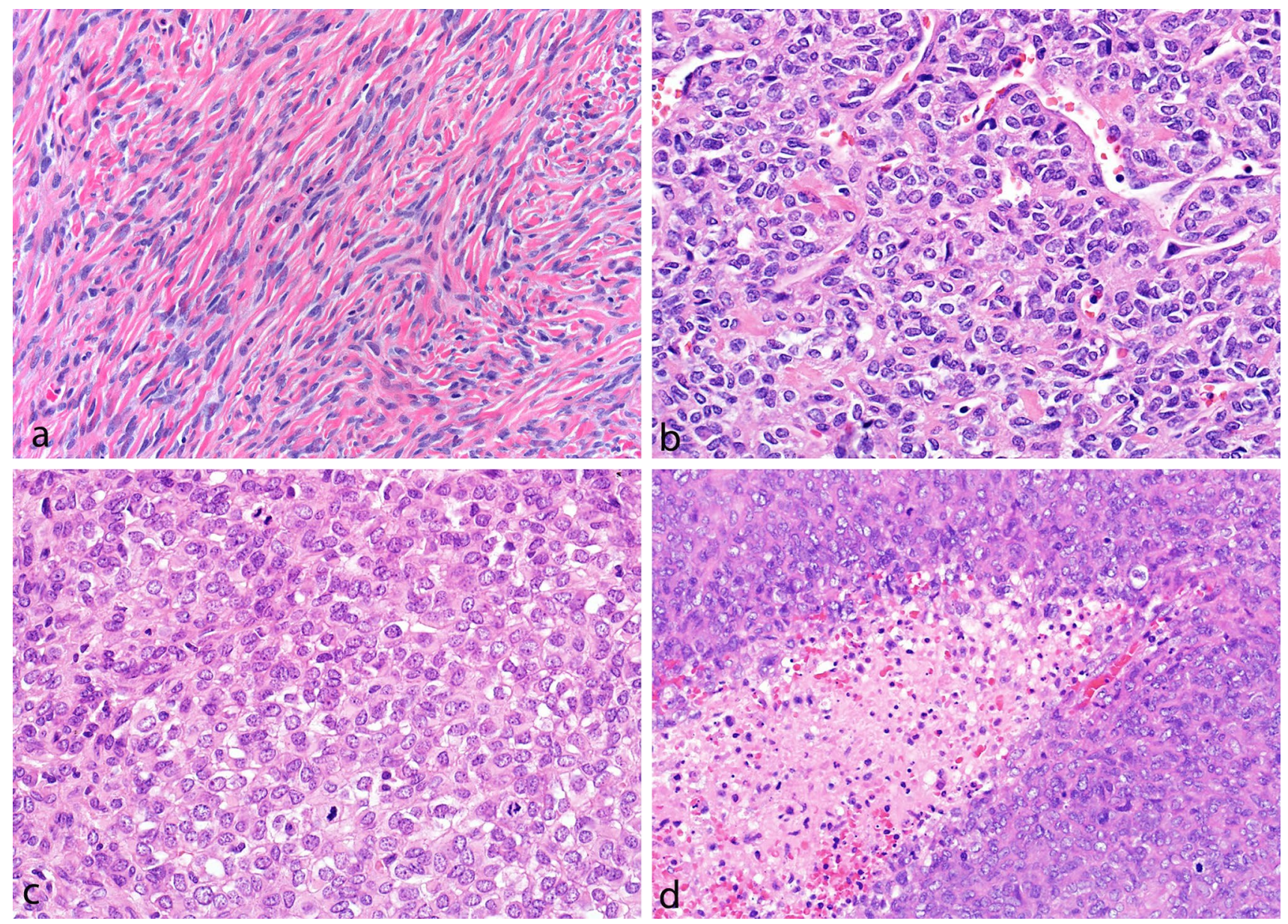

Fig. 1 Tumors classified as solitary fibrous tumor contained uniform ovoid-to-slightly spindled-shaped cell deposited in a collagenized background and arranged around branching and hyalinized blood vessels (a), while hemangiopericytomas were highly cellular tumors

Grading of Solitary Fibrous Tumor/Hemangiopericytoma

\begin{tabular}{|c|c|c|c|c|}
\hline \multirow[b]{2}{*}{ Phenotype } & \multirow[b]{2}{*}{ Mitoses } & \multicolumn{3}{|c|}{ WHO } \\
\hline & & & VS 2016 & Soft Tissue 2 \\
\hline SFT & $+<5$ & $=$ & $\begin{array}{c}1 \\
N=43\end{array}$ & \\
\hline $\begin{array}{l}\text { Intermediate } \\
\text { HPC }\end{array}$ & $+<5$ & $=$ & $\begin{array}{c}2 \\
N=41\end{array}$ & \\
\hline $\begin{array}{l}\text { SFT } \\
\text { Intermediate } \\
\text { HPC }\end{array}$ & $+\quad \geq 5$ & $=$ & $\begin{array}{c}3 \\
N=49\end{array}$ & $\begin{array}{c}\text { Malignant } \\
\text { SFT } \\
\mathrm{N}=49\end{array}$ \\
\hline
\end{tabular}

Fig. 2 CNS (2016) and Soft Tissue (2013) WHO Classification schemes

Inc, Boulder, CO, USA) to detect fusions among 26 genes employing the Anchored Multiplex PCR-based enrichment, identifying NAB2-STAT6 gene fusions in an additional 47 composed of predominantly round cells in a sheet-like pattern (b) with less prominent vasculature often showing high mitotic rates (c) and necrosis $(\mathbf{d})$

samples. Briefly, a total of up to 250 ng RNA were used as starting material. Library preparation was performed according to the manufacturer's protocol (ArcherDx, Inc) and sequencing was done on a NextSeq550 instrument using NextSeq500/550 High Output v2 kit (150 cycles) (Illumina, Inc., San Diego, CA, USA). The resulting raw data were converted to fastq files and were then processed with the automated Archer Analysis Bioinformatics Platform (Version Archer Analysis 5.1, ArcherDx, Inc).

\section{Statistical methods}

Patient and tumor characteristics were summarized with frequencies and percentages or medians, interquartile ranges (IQR), or ranges, as appropriate. Fisher's exact tests were used to compare categorical variables between selected groups (e.g., CNS-G, ST-G, and molecular cluster type). Our correlative analysis was based on the time of surgery, which represents when the first tumor was evaluated at the 
institution. This was the primary tumor resection for 97 patients, resection of a recurrent tumor for 35 patients, and of a metastasis for 1 patient. This approach was made necessary by the fact that, in 36 patients, the primary tumor was not available, and no assumption could be made regarding the grade of the primary tumor. A recent study has shown histological progression of tumor at recurrence in $16 \%$ of cases [2]. Recurrence-free survival (RFS) was defined as the time between surgery and the first adverse event (local recurrence or metastasis) following surgery, censoring patients with no adverse event at time of last follow-up. Overall survival (OS) was defined as the time between surgery and death (any cause), censoring those still alive at last followup. OS and RFS were estimated using the Kaplan-Meier method, and were summarized at 5, 10, and 20-year postsurgery, along with the median survival. OS and RFS were compared between selected groups with Cox proportionalhazard regression models, using the likelihood ratio test to assess significance. $95 \%$ confidence intervals (CI) were also reported for the survival estimates and hazard ratios (HR). We also investigated RFS from time of original diagnosis to the first adverse event in the patient's history (this also considers the time interval prior to when a recurrent sample was evaluated), as well as OS from time of original diagnosis. For disease-free survival, deaths due to disease were the events, and all non-events (deaths with unknown or other causes, along with those still alive) were censored. All analyses were performed using SAS version 9.4 (SAS Institute Inc, Cary, NC, USA), or R [19]. $p$ values less than 0.05 were considered statistically significant.

\section{Results}

Table 1 summarizes the characteristics of the patient cohort.

\section{Clinical outcome}

Follow-up was available in 129 of 133 patients (range 4 days-22.2 years; median 5 years). At last follow-up, 104 patients were alive (47 without disease, 24 with disease, and 33 alive but their disease status was unknown) and 29 patients were deceased ( 20 of disease, 6 of other causes, and 3 of unknown cause). The median recurrence-free (RFS) and overall survival (OS) times from the original diagnosis were 9.6 years and 20.9 years, respectively (Fig. 3). When RFS and OS were calculated from the time at which the first surgical specimen was available for pathology review (indicated as time of surgery), the median RFS and OS were 11.3 years and 14.7 years, respectively. The differences in RFS and OS with respect to time of diagnosis versus time of surgery are explained by the influence of the patients in which the first available specimen was a recurrence rather than a primary tumor, as illustrated in suppl. Figure 1 and suppl. Figure 2 (Online Resource 1 and Online Resource 2). When considering only cases in which the primary tumor was available for review, the median RFS for primary tumors was 12.9 years ( $79.0 \%$ at 5 years) compared to 7.8 years $(67.6 \%$ at 5 years) for recurrent cases $(p=0.14)$. The median OS from the time of surgery for cases in which the primary tumor was available for review was 14.7 years $(89.1 \%$ at 5 years) compared to 9.6 years ( $70.3 \%$ at 5 years) for those in which the recurrence was the first available material $(p=0.03)$. The disease-free survival for all patients at 5 years was $87.2 \%(95 \%$ CI $80.3,94.1)$ and at 10 years was $74.2 \%$ (95\% CI 62.6, 85.7). For patients in which the primary tumor was reviewed, the 5- and 10-year survivals were $91.5 \%$ (95\% CI 84.9, 98.1) and 85.5\% (95\% CI 75.2, 95.7), respectively, while the 5- and 10-year survivals for patients in which a recurrence was reviewed was $85.5 \%$ (95\% CI 75.2, 95.7) and 43.8\% (16.3, 71.3), respectively.

Of the entire cohort, 42 patients experienced at least one adverse event after surgery (local recurrence or metastasis): 7 patients had metastases only, 33 had local recurrence only, and 2 patients had both local recurrence and metastases (Table 2). When looking only at the patients from whom the primary tumor was reviewed ( $n=97), 28$ patients experienced either recurrence or metastasis: 4 patients had metastases only, 22 patients had recurrence only, and 2 patients had both recurrence and metastases.

Among those in the full cohort who experienced an adverse event, the median time from original diagnosis to the first local recurrence was 5.6 years (range 27 days -36.7 years), while the median time to first metastasis was 8.8 years (range 7.1 months-23.4 years). For patients from whom the primary tumor was reviewed, the median time to first recurrence was 3.5 years (range 27 days-14.9 years) with median time to first metastasis of 4.5 years (range 7.1 months-10.3 years).

\section{Histopathology}

By histologic criteria, 55 tumors were classified as SFT phenotype, 24 as HPC phenotype, and the remaining 54 were considered intermediate. The median mitotic rate was $2(\times 10 \mathrm{HPF})(\mathrm{IQR} 1-6$; range $0-45$ mitoses per 10 HPF). Necrosis was identified in 16 cases (12\%), while 117 tumors lacked this finding. On univariate analysis, mitotic rate $(\geq 5$ mitoses $\times 10 \mathrm{HPF})$ and necrosis were both significantly associated with recurrence-free survival (RFS, $p=0.004, p=0.002$, respectively) but not overall survival (OS, $p=0.12, p=0.54$ ) (Tables 3, 4). Five- and ten-year RFS were $51.1 \%$ and $0 \%$, respectively, for tumors with necrosis and $80.1 \%$ and $56 \%$ for those without necrosis (Table 3). 
Table 1 Clinicopathologic features

\begin{tabular}{|c|c|c|c|c|}
\hline & \multirow[t]{2}{*}{ Total $(N=133)^{\mathrm{a}}$} & \multicolumn{3}{|c|}{ Specimen type at time of surgery } \\
\hline & & Primary $(N=97)^{\mathrm{a}}$ & Recurrence $(N=35)^{\mathrm{a}}$ & Metastasis $(N=1)^{\mathrm{a}}$ \\
\hline \multicolumn{5}{|l|}{ Age at surgery } \\
\hline Median & 54.1 & 54.1 & 55.3 & 41.4 \\
\hline Range & $(20.1-87.3)$ & $(20.1-83.0)$ & $(39.0-87.3)$ & $(41.4-41.4)$ \\
\hline \multicolumn{5}{|l|}{ Age at initial diagnosis } \\
\hline Median & 49.2 & 52.9 & 39.3 & \\
\hline Range & $(17.5-78.8)$ & $(20.1-78.8)$ & $(17.5-61.1)$ & \\
\hline \multicolumn{5}{|l|}{ Sex } \\
\hline Female $(\%)$ & $59(44.4)$ & $42(43.3)$ & $17(48.6)$ & 0 \\
\hline Male & $74(55.6)$ & $55(56.7)$ & $18(51.4)$ & 1 \\
\hline \multicolumn{5}{|l|}{ Race/ethnicity } \\
\hline Caucasian $(\%)$ & $103(77.4)$ & $77(79.4)$ & $26(74.3)$ & 0 \\
\hline Hispanic/Latino & $6(4.5)$ & $5(5.2)$ & $1(2.9)$ & 0 \\
\hline African American & $9(6.8)$ & $7(7.2)$ & $2(5.7)$ & 0 \\
\hline Native American & $2(1.5)$ & $0(0)$ & $2(5.7)$ & 0 \\
\hline Asian & $3(2.3)$ & $3(3.1)$ & $0(0)$ & 0 \\
\hline Pacific Islander & $2(1.5)$ & $1(1.0)$ & $1(2.9)$ & 0 \\
\hline Other & $2(1.5)$ & $1(1.0)$ & $1(2.9)$ & 0 \\
\hline Unknown & $6(4.5)$ & $3(3.1)$ & $2(5.7)$ & 1 \\
\hline \multicolumn{5}{|l|}{ Phenotype } \\
\hline HPC (\%) & $24(18.0)$ & $15(15.5)$ & $9(25.7)$ & 0 \\
\hline INT & $54(40.6)$ & $39(40.2)$ & $14(40.0)$ & 1 \\
\hline SFT & $55(41.4)$ & $43(44.3)$ & $12(34.3)$ & 0 \\
\hline \multicolumn{5}{|l|}{ Tumor size $(\mathrm{cm})$} \\
\hline$N$ & 96 & 75 & 21 & 0 \\
\hline Median & 4.1 & 4.5 & 3.8 & \\
\hline Q1, Q3 & $3.3,6.0$ & $3.1,6.5$ & $3.3,5.0$ & \\
\hline Range & $(1.3-11.0)$ & $(1.3-11.0)$ & $(1.4-6.5)$ & \\
\hline \multicolumn{5}{|l|}{ Mitoses (/10 hpf) } \\
\hline Median & 2.0 & 1.0 & 3.0 & 16.0 \\
\hline Q1, Q3 & $1.0,6.0$ & $1.0,6.0$ & $1.0,8.0$ & \\
\hline Range & $(0.0-45.0)$ & $(0.0-36.0)$ & $(0.0-45.0)$ & \\
\hline \multicolumn{5}{|l|}{ Necrosis } \\
\hline Absent (\%) & $117(88.0)$ & $87(89.7)$ & $29(82.9)$ & 1 \\
\hline Present & $16(12.0)$ & $10(10.3)$ & $6(17.1)$ & 0 \\
\hline \multicolumn{5}{|l|}{ CNS-G grade } \\
\hline $1(\%)$ & $43(32.3)$ & $36(37.1)$ & $7(20.0)$ & 0 \\
\hline 2 & $41(30.8)$ & $28(28.9)$ & $13(37.1)$ & 0 \\
\hline 3 & $49(36.8)$ & $33(34.0)$ & $15(42.9)$ & 1 \\
\hline \multicolumn{5}{|l|}{ ST-G grade } \\
\hline Low $(\%)$ & $84(63.2)$ & $64(66.0)$ & $20(57.1)$ & 0 \\
\hline High & $49(36.8)$ & $33(34.0)$ & $15(42.9)$ & 1 \\
\hline \multicolumn{5}{|l|}{ Extent of resection } \\
\hline Gross total resection (\%) & $63(56.3)$ & $55(62.5)$ & $8(33.3)$ & 0 \\
\hline Subtotal resection & $49(43.8)$ & $33(37.5)$ & $16(66.7)$ & 0 \\
\hline \multicolumn{5}{|l|}{ CD34 } \\
\hline Negative $(<5)(\%)$ & $25(18.8)$ & 19 (19.6) & $6(17.1)$ & 0 \\
\hline Focal (5-50) & $32(24.1)$ & $21(21.6)$ & $10(28.6)$ & 1 \\
\hline Diffuse $(>50)$ & $76(57.1)$ & $57(58.8)$ & $19(54.3)$ & 0 \\
\hline
\end{tabular}


Table 1 (continued)

Total $(N=133)^{\mathrm{a}}$

Specimen type at time of surgery

\begin{tabular}{lll}
\hline Primary $(N=97)^{\mathrm{a}}$ & Recurrence $(N=35)^{\mathrm{a}}$ & Metastasis $(N=1)^{\mathrm{a}}$
\end{tabular}

Treatment

Radiation only (\%)

$60(53.1)$

$50(58.1)$

$10(37.0)$

0

Chemotherapy only

$1(0.9)$

$1(1.2)$

$0(0.0)$

Radiation + chemotherapy

$3(2.7)$

$2(2.3)$

No treatment

49 (43.4)

$33(38.4)$

$1(3.7)$

$16(59.3)$

0

Molecular cluster

ex2-3_ex18/ex2_ex1-2/other (\%)

$10(7.5) \quad 8(8.2)$

ex4_ex2-3

$29(21.8)$

$22(22.7)$

$60(45.1)$

$46(47.4)$

No fusion detected

$12(9.0)$

$8(8.2)$

Failed or unavailable

22 (16.5)

$13(13.4)$

2 (5.7) 0

$7(20.0) \quad 0$

$14(40.0) \quad 0$

$3(8.6) \quad 1$

9 (25.7)

${ }^{\mathrm{a}}$ Frequencies not summing to column total indicate missing data. For continuous variables, $\mathrm{N}$ is provided in cases of missing data

\section{Outcome from the Time of Original Diagnosis}

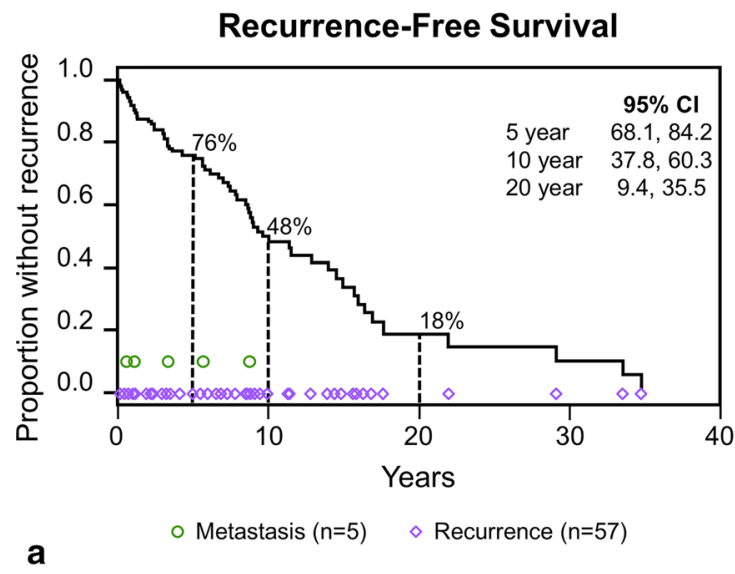

Fig. 3 Kaplan-Meier plots showing the median recurrence-free survival (RFS) and overall survival (OS) from the time of the original diagnosis, 9.6 years and 20.9 years, respectively. Note that there was

\section{Tumor size}

Tumor size was available in 96 patients ranging from 1.3 to $11.0 \mathrm{~cm}$ (Table 1). Tumor size was not significantly associated with RFS or OS when considered continuously or categorically (tumor size $<5 \mathrm{~cm}$ vs tumor size $\geq 5 \mathrm{~cm}$ ) (Tables 3, 4).

\section{Immunohistochemistry}

CD34 was recorded as diffuse in the majority of cases $(n=76 ; 57 \%)$, while 32 cases $(24 \%)$ were focally positive and 25 were negative (19\%). STAT6 immunostain showed nuclear expression in 132 (99\%) cases. The single case lacking nuclear expression of STAT6 was a T11 spinal mass
Overall Survival

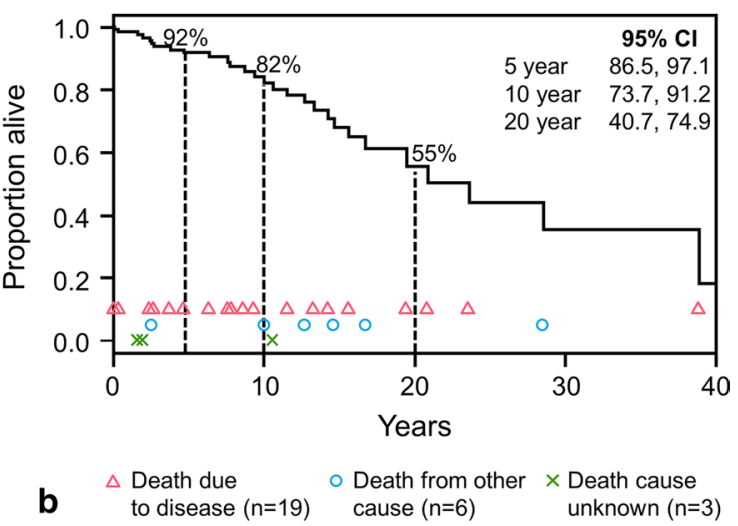

one death due to disease with unknown date of death and that patient is not included in these plots

from a 40-year-old woman that recurred multiple times. STAT6 immunochemistry was performed on two separate specimens from this patient and repeated at two different centers (Mayo Clinic and the originating institution), and all samples tested were negative. Both specimens of this tumor were tested by PCR and showed an NAB2 exon 6-STAT6 exon 16 fusion.

\section{Grading}

Tumors were classified as grade $1(n=43), 2(n=41)$, or 3 ( $n=49)$ using the CNS-G, and SFT $(n=84)$ or malignant SFT ( $n=49)$ using the ST-G scheme (Fig. 2). When comparing the extent of CD34 immunostaining among those with at least 5\% staining, tumors with SFT phenotype were 
Table 2 Recurrence and metastasis since original diagnosis

\begin{tabular}{lll}
\hline & \multicolumn{2}{l}{$N=133$ Patients } \\
\cline { 2 - 3 } & Recurrence & Metastasis \\
\hline \# Of events per patient & 74 & 116 \\
0 & 36 & 15 \\
1 & 13 & 2 \\
2 & 10 & 0 \\
$3+$ & 34 & 8 \\
First event after surgery & 28 metastases from 17 \\
Metastasis since diagnosis, site ${ }^{\mathrm{a}}$ & patients \\
& 7 & \\
Bone (includes sternum) & 7 \\
Liver & 7 \\
Lung & 2 \\
Brain & 1 \\
Kidney & 1 \\
Pancreas & 3 \\
Other (includes CSF, adnexa, epi- & \\
$\quad$ dural) &
\end{tabular}

a 17 patients had at least one metastasis (five of these patients' metastasis was in multiple sites). Two of these patients had two metastases (brain followed by brain; bone followed by epidural)

more likely to show diffuse CD34 immunoreactivity (88\%) as compared to tumors with HPC and INT phenotype (35\% and $63 \%$, respectively), $p=0.0001$. Furthermore, CNS-G 1 tumors were more likely to show diffuse CD34 immunoreactivity (85\%) as compared to CNS-G $2(56 \%)$ or $3(68 \%)$, $p=0.03$.

On univariate analysis, both the CNS-G and ST-G were significantly associated with RFS (Table $3, p=0.014,0.004$, respectively) (Fig. 4a, b) but not overall survival ( $p=0.23$, $p=0.12$, respectively). Five-year RFS rates were, respectively, 82,88 , and $60 \%$, while 10 -year rates were 61,58 , and $34 \%$ for CNS grades 1, 2, and 3. They were 85 and 60 at 5 years, and 59 and $34 \%$ at 10 years for SFT and malignant SFT, respectively. When considering only the primary tumors, ST-G remained significant $(p=0.03)$, while the CNS-G did not $(p=0.07)$. When evaluating only the recurrent cases, the ST-G approached significance $(p=0.05)$, while the CNS-G did not $(p=0.15)$. Since the ST-G scheme appeared to be a simpler way to stratify these tumors, and necrosis was associated with decreased RFS on univariate analysis, we attempted to incorporate necrosis into the ST-G and stratify tumors into three tiers: (1) tumors with $<5$ mitoses/10 HPF, (2) tumors with $\geq 5$ mitoses/10 HPF without necrosis, and (3) tumors with $\geq 5$ mitoses/10 HPF with necrosis. This modified soft-tissue grading scheme showed a strong association with recurrence-free survival $(p=0.0006)$ and remained significant $(p=0.02)$ when considering only the primary tumors and only recurrent tumors $(p=0.04)$ (Fig. 5a-c). Among 84 tumors with $<5$ mitoses per $10 \mathrm{HPF}$, necrosis was present only in 3 (3.6\%). All three tumors had a hemangiopericytomatous-like phenotype. In two patients, necrosis was present at the time of primary surgery. One of these patients had GTR and one STR. Both received radiation therapy. They were both alive without recurrence, respectively, at 67 and 38 months. In the third patient, we reviewed a recurrent sample, 16 years and 3 months from the original diagnosis. Only 1 month postoperative follow-up was available in this patient.

\section{NAB2-STAT6 fusion}

Molecular analysis was successful in 111 (of 127; 87\%) cases which could be tested, and NAB2-STAT6 fusion was detected in 99 (of $111 ; 89 \%$ ) successfully tested tumors: $N A B 2$ exon 5-7-STAT6 exon 16-17 $(n=60), N A B 2$ exon 4-STAT6 exon 2-3 $(n=29), N A B 2$ exon 2-STAT6 exon 1-2 $(n=4), N A B 2$ exon 2-3-STAT6 exon $18(n=4), N A B 2$ exon 2-STAT6 exon $5(n=1)$, and NAB2 exon 7-STAT6 exon $1(n=1)$. The remaining 12 cases lacked an identifiable NAB2-STAT6 fusion. Fusion variants were grouped according to their frequency and predicted protein domain inclusion into four groups. The four groups based on fusion variant included: (1) NAB2 exon 4-STAT6 exon 2-3 $(n=29)$, (2) NAB2 exon 5-7-STAT6 exon 16-17 $(n=60)$, (3) other NAB2-STAT6 fusions ( $n=10)$, and (4) no detectable fusion $(n=12)$. Figure 6 illustrates the relationship between the four NAB2-STAT6 fusion groups, and phenotype, mitoses, necrosis, CNS-G, ST-G, and modified ST-G.

Fusion cluster and phenotype When comparing fusion cluster with phenotype, the majority of NAB2 exon 4-STAT6 exon 2-3 fusions were seen among tumors with an SFT phenotype ( $n=24$ of $29,83 \%$ ), with four cases showing intermediate phenotype and one with HPC phenotype. The tumors which harbored NAB2 exon 5-7-STAT6 exon 16-17 were more likely to have an intermediate or HPC phenotype ( $n=49$ of 60 and $82 \% ; n=34$, and $n=15$, respectively) $(p<0.0001)$, the remaining $11(18 \%)$ showing an SFT phenotype. Among the ten with other fusions, half (5) were SFT, four intermediate, and one HPC. Among the 12 with no fusion detected, 4 were SFT, 6 intermediate, and 2 HPC.

Fusion cluster and mitotic rate Tumors with NAB2 exon 4-STAT6 exon 2-3 or "other fusions" were more likely to have mitotic rates $<5 / 10 \mathrm{HPF}$, whereas tumors with NAB2-STAT6 5-7-STAT6 exon 16-17 and without a detectable fusion were more likely to have mitotic rates $\geq 5 / 10$ $\operatorname{HPF}(p=0.002)$.

Fusion cluster and necrosis There was no significant association between fusion type or status (absence or presence of a detected fusion) and necrosis ( $p=0.16 ; p=0.35$, respectively).

Fusion cluster and CNS-G There was a statistically significant association between type of fusion and CNS-G 
Table 3 Recurrence-free survival (RFS)

\begin{tabular}{|c|c|c|c|c|c|c|c|}
\hline Variable & $N$ & Events & $\begin{array}{l}\text { Median years (95\% } \\
\text { CI) }{ }^{\mathrm{a}}\end{array}$ & $\begin{array}{l}5 \text {-Year RFS \% (95\% } \\
\text { CI) })^{\mathrm{a}}\end{array}$ & $\begin{array}{l}\text { 10-Year RFS (95\% } \\
\text { CI) })^{\mathrm{a}}\end{array}$ & Hazard ratio $(95 \% \mathrm{CI})$ & $p$ value \\
\hline \multicolumn{7}{|l|}{ Phenotype } & 0.87 \\
\hline HPC & 24 & 7 & 7.8 (5.7-NA) & $81.1 \%(64.2 \%, 97.9 \%)$ & $41.7 \%(8.2 \%, 75.2 \%)$ & (Reference) & \\
\hline INT & 54 & 18 & 12.9 (8.8-NA) & $78.8 \%(66.3 \%, 91.3 \%)$ & $52.7 \%(34.6 \%, 70.8 \%)$ & $0.88(0.38,2.30)$ & \\
\hline SFT & 55 & 17 & 11.3 (6.1-NA) & $71.3 \%(57.4 \%, 85.3 \%)$ & $53.1 \%(34.4 \%, 71.9 \%)$ & $1.06(0.45,2.74)$ & \\
\hline \multicolumn{7}{|l|}{ Mitoses } & 0.004 \\
\hline$<5$ & 84 & 21 & 14.9 (8.9-NA) & $85.0 \%(76.4 \%, 93.7 \%)$ & $58.7 \%(42.8 \%, 74.6 \%)$ & (Reference) & \\
\hline$\geq 5$ & 49 & 21 & $5.7(3.0-12.9)$ & $59.6 \%(43.3 \%, 76.0 \%)$ & $33.6 \%(13.9 \%, 53.2 \%)$ & $2.48(1.34,4.59)$ & \\
\hline \multicolumn{7}{|l|}{ Necrosis } & 0.002 \\
\hline Absent & 117 & 33 & 12.9 (8.2-NA) & $80.1 \%(71.8 \%, 88.5 \%)$ & $55.7 \%(42.5 \%, 68.9 \%)$ & (Reference) & \\
\hline Present & 16 & 9 & $5.6(1.5-8.8)$ & $51.1 \%(25.0 \%, 77.3 \%)$ & $0.0 \%(0.0 \%, 0.0 \%)$ & $4.04(1.76,8.51)$ & \\
\hline \multicolumn{7}{|l|}{ Tumor size } & 0.46 \\
\hline $0-5 \mathrm{~cm}$ & 52 & 12 & & $78.1 \%(65.3 \%, 90.9 \%)$ & $61.6 \%(41.9 \%, 81.4 \%)$ & - & \\
\hline$\geq 5 \mathrm{~cm}$ & 44 & 18 & $8.8(5.6-)$ & $71.3 \%(56.9 \%, 85.8 \%)$ & $46.2 \%(27.4 \%, 65.1 \%)$ & $1.31(0.64,2.80)$ & \\
\hline \multicolumn{7}{|l|}{ CNS grade (2016) } & 0.01 \\
\hline 1 & 43 & 11 & NA & $81.9 \%(68.6 \%, 95.1 \%)$ & $61.0 \%(40.5 \%, 81.5 \%)$ & (Reference) & \\
\hline 2 & 41 & 10 & 14.9 (8.9-NA) & $88.2 \%(77.3 \%, 99.2 \%)$ & $58.3 \%(35.3 \%, 81.2 \%)$ & $0.80(0.33,1.90)$ & \\
\hline 3 & 49 & 21 & $5.7(3.0-12.9)$ & $59.6 \%(43.3 \%, 76.0 \%)$ & $33.6 \%(13.9 \%, 53.2 \%)$ & $2.21(1.08,4.78)$ & \\
\hline \multicolumn{7}{|l|}{ Soft-tissue grade (2013) } & 0.004 \\
\hline SFT & 84 & 21 & 14.9 (8.9-NA) & $85.0 \%(76.4 \%, 93.7 \%)$ & $58.7 \%(42.8 \%, 74.6 \%)$ & (Reference) & \\
\hline Malignant SFT & 49 & 21 & $5.7(3.0-12.9)$ & $59.6 \%(43.3 \%, 76.0 \%)$ & $33.6 \%(13.9 \%, 53.2 \%)$ & $2.48(1.34,4.59)$ & \\
\hline \multicolumn{7}{|c|}{ Modified soft-tissue grade } & 0.0006 \\
\hline SFT & 84 & 21 & $14.9(8.9-)$ & $85.0 \%(76.4 \%, 93.7 \%)$ & $58.7 \%(42.8 \%, 74.6 \%)$ & (Reference) & \\
\hline $\begin{array}{l}\text { Malignant SFT no } \\
\text { necrosis }\end{array}$ & 36 & 12 & $7.8(3.3-)$ & $68.1 \%(49.2 \%, 87.0 \%)$ & $46.7 \%(22.7 \%, 70.7 \%)$ & $1.75(0.83,3.52)$ & \\
\hline $\begin{array}{l}\text { Malignant SFT with } \\
\text { necrosis }\end{array}$ & 13 & 9 & $2.0(1.2-8.8)$ & $42.7 \%(14.7 \%, 70.8 \%)$ & Not estimable* & $6.03(2.53,13.34)$ & \\
\hline \multicolumn{7}{|l|}{ Resection extent } & 0.27 \\
\hline Gross total & 63 & 18 & 12.9 (8.8-NA) & $79.1 \%(67.4 \%, 90.8 \%)$ & $60.0 \%(42.5 \%, 77.5 \%)$ & (Reference) & \\
\hline Subtotal & 49 & 19 & 7.8 (5.7-NA) & $69.6 \%(55.7 \%, 83.5 \%)$ & $42.6 \%(23.5 \%, 61.6 \%)$ & $1.44(0.75,2.77)$ & \\
\hline \multicolumn{7}{|l|}{ Radiation } & 0.79 \\
\hline No & 50 & 16 & $9.2(7.8-\mathrm{NA})$ & $75.5 \%(62.1 \%, 88.8 \%)$ & $48.9 \%(27.4 \%, 70.3 \%)$ & (Reference) & \\
\hline Yes & 63 & 20 & $12.9(6.1-\mathrm{NA})$ & $78.5 \%(67.0 \%, 89.9 \%)$ & $50.8 \%(32.2 \%, 69.3 \%)$ & $0.91(0.47,1.80)$ & \\
\hline \multicolumn{7}{|c|}{ Molecular cluster (most common types) } & 0.80 \\
\hline ex4_ex2-3 & 29 & 9 & $11.3(3.3-\mathrm{NA})$ & $68.2 \%(48.6 \%, 87.8 \%)$ & $59.7 \%(36.5 \%, 82.9 \%)$ & (Reference) & \\
\hline ex5-7_ex16-17 & 60 & 22 & $8.8(6.4-\mathrm{NA})$ & $74.0 \%(61.7 \%, 86.4 \%)$ & $42.7 \%(25.6 \%, 59.9 \%)$ & $1.03(0.49,2.36)$ & \\
\hline No fusion detected & 12 & 4 & $12.9(1.3-\mathrm{NA})$ & $\begin{array}{l}90.0 \%(71.4 \% \\
100.0 \%)\end{array}$ & $\begin{array}{l}72.0 \%(37.1 \% \\
100.0 \%)\end{array}$ & $0.72(0.19,2.26)$ & \\
\hline \multicolumn{7}{|l|}{ Fusion status } & 0.52 \\
\hline No fusion detected & 12 & 4 & $12.9(1.3-\mathrm{NA})$ & $\begin{array}{l}90.0 \%(71.4 \% \\
100.0 \%)\end{array}$ & $\begin{array}{l}72.0 \%(37.1 \% \\
100.0 \%)\end{array}$ & (Reference) & \\
\hline $\begin{array}{l}\text { Fusion detected (any } \\
\text { type) }\end{array}$ & 99 & 33 & 8.9 (6.9-NA) & $73.0 \%(62.9 \%, 83.0 \%)$ & $46.5 \%(32.2 \%, 60.7 \%)$ & $1.39(0.54,4.74)$ & \\
\hline
\end{tabular}

${ }^{a} \mathrm{NA}$ indicates that the value was not able to be estimated

( $p<0.0001)$. The 29 tumors with NAB2 exon 4-STAT6 exon 2-3 were more frequently grade 1 tumors $(n=21)$ than grade 2 or 3 ( $n=3, n=5$, respectively). The 60 tumors with $N A B 2$ exon 5-7-STAT6 exon 16-17 fusion were more frequently grade 2 and 3 tumors ( $n=22, n=31$, respectively).
Fusion cluster and $S T-G$ There was a statistically significant association between type of fusion and ST-G ( $p=0.002$ ). The 29 tumors harboring NAB2 exon 4-STAT6 exon 2-3 or the 10 with "other fusions" were more likely to be solitary fibrous tumors $(n=24, n=8)$ compared to 
Table 4 Overall survival (OS)

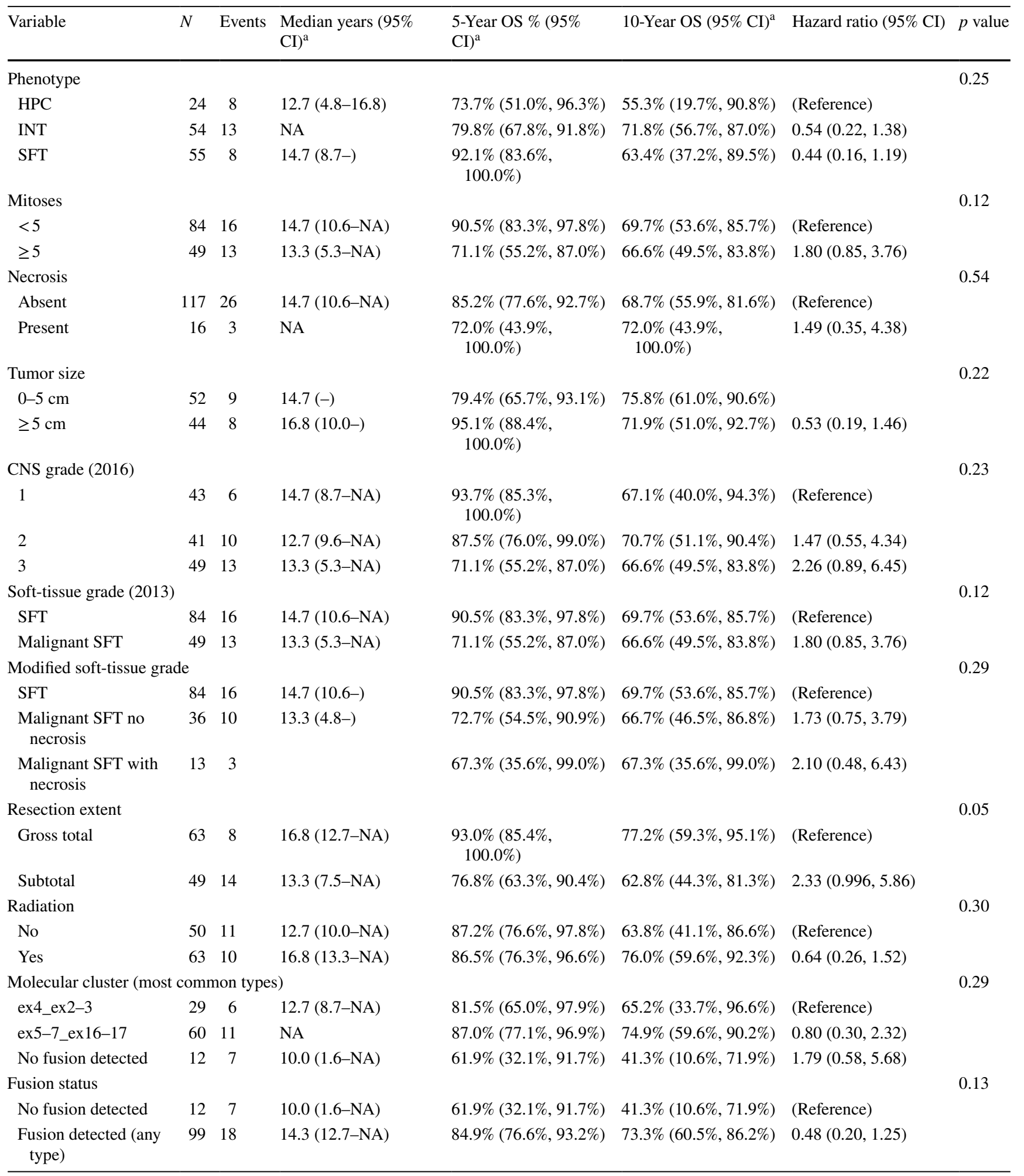

${ }^{a} \mathrm{NA}$ indicates that the value was not able to be estimated

malignant solitary fibrous tumors $(n=5, n=2)$. The 60 tumors with NAB2 exon 5-7-STAT6 exon 16-17 were malignant SFT in $31(52 \%)$ and SFT in $29(48 \%)$. The majority of tumors without a detectable fusion were malignant SFT $(n=8)$ compared to SFT $(n=4)$.

Fusion cluster and outcome NAB2-STAT6 fusion type was not associated with RFS $(p=0.80)$ or OS $(p=0.29)$. 


\section{Recurrence-Free Survival from the Time of Surgery}

CNS WHO Grade 2016

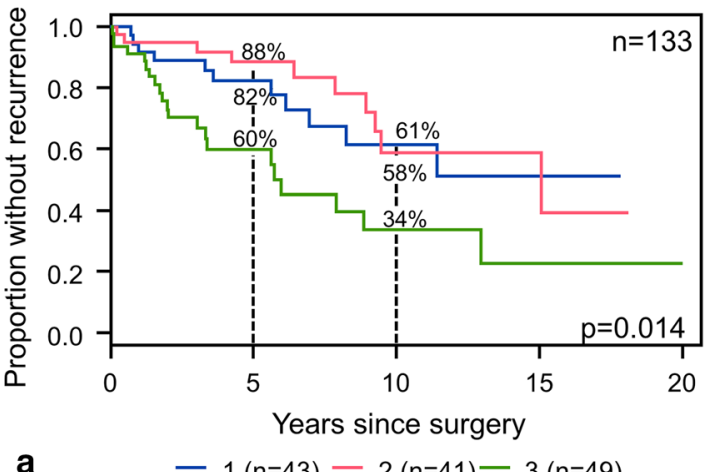

Soft Tissue WHO Grade 2013

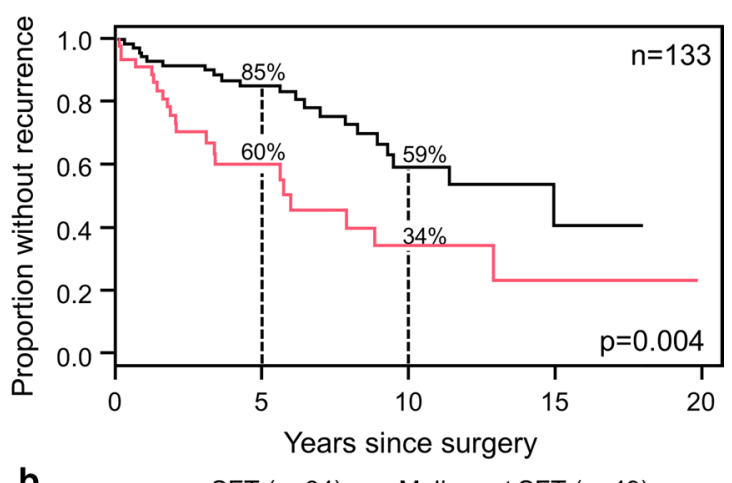

Fig. 4 Univariate analysis shows that both the CNS-G (a) and ST-G (b) are significantly associated with recurrence-free survival (Table 3, $p=0.01,0.004$, respectively) but not overall survival ( $p=0.23, p=0.12$, respectively)

Fig. 5 Modified soft-tissue grading scheme showed strong association with recurrencefree survival $(p=0.0006)$ and remained significant $(p=0.02)$ when considering only the primary tumors and only recurrent tumors $(p=0.04)(\mathbf{a}-\mathbf{c})$
Fig. 6 This graph highlights the relationship between the four NAB2-STAT6 fusion groups, and phenotype, mitoses, necrosis, CNS-G, ST-G, and modified ST-G
Recurrence-Free Survival by Soft Tissue WHO Grade (2013) and Necrosis From the Time of Surgery
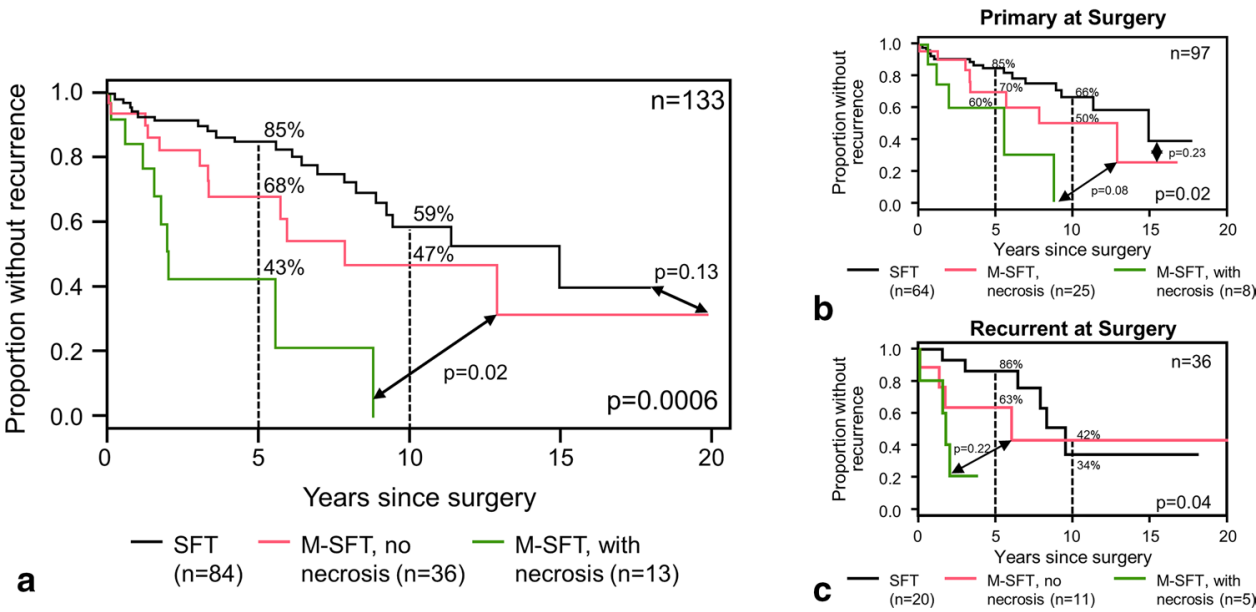

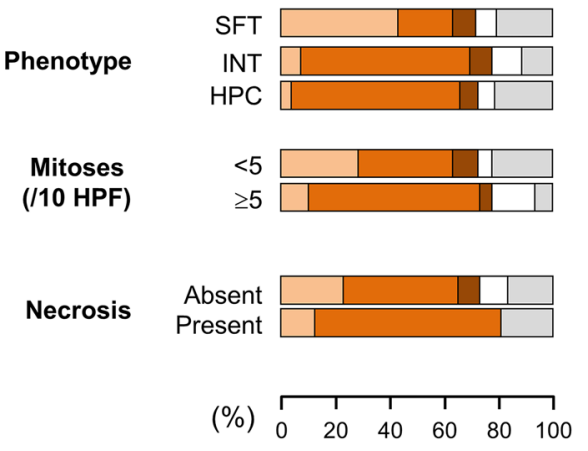

CNS-Grade

ST-Grade

Modified SFT

Malignant SFT

Malignant SFT with Necrosis

(\%) \begin{tabular}{lllllll}
\hline & 20 & 40 & 60 & 80 & 100
\end{tabular}

$\square$ ex4 ex 2-3 $\square$ ex 5-7 ex 16-17

Other fusions

No fusion $\square$ Not done/failed 
Furthermore, when comparing tumors with and without identifiable NAB2-STAT6 fusions (dichotomously), there was no significant difference in recurrence-free survival $(p=0.52)$ or overall survival $(p=0.13)$.

\section{Treatment data}

Sixty-three patients $(56.3 \%)$ underwent gross total resection, while the tumor was subtotally resected in 49 patients (43.8\%). In the remaining 21 patients, the extent of surgical resection was unknown. Sixty patients $(53.1 \%)$ received only adjuvant radiation therapy, a single patient $(1 \%)$ was treated with chemotherapy alone, 3 patients $(2.7 \%)$ underwent both adjuvant radiation and chemotherapy, and the remaining 49 patients $(43.4 \%)$ were treated with surgery alone. Neither extent of resection nor adjuvant radiation therapy was significantly associated with outcome (overall survival: $p=0.051$, 0.30 ; recurrence-free survival: $p=0.27,0.79$, respectively).

\section{Discussion}

Our study confirms the natural history of meningeal-based SFT/HPC, showing high rates of local recurrence and distant metastasis. Furthermore, patients in our cohort demonstrate a tendency for late adverse events, a subset developing their first recurrences/metastasis over 3 decades after the initial diagnosis. Similarly, patients continue to die of disease almost 40 years after the initial diagnosis. In a subset of our cases, only material from recurrence (not the primary tumor) was available for review. As these patients are at greater risk for adverse events, as shown by our data, these cases influence the OS and RFS of the entire cohort. However, trends for overall survival and RFS of both primary and recurrent cases remain similar.

On univariate analysis, mitotic rate and necrosis were each significantly associated with RFS but not OS. Since the CNS-G and ST-G schemes take mitotic rate into account, it is not surprising that both appear to show association with RFS. However, there does not appear to be a significant difference between grade 1 and grade 2 according to CNS-G, and the ST-G scheme, based solely on mitotic rate, appears to stratify tumors in a simpler and more efficient fashion. Furthermore, the ST-G maintains statistical significance when looking only at primary cases. A recent risk stratification model proposed by Demicco et al. for non-meningeal tumors incorporates mitotic rate and necrosis, along with patient age and tumor size, and appears to show prognostic significance in determining propensity for metastatic disease [8]. The class sizes used in soft tissue with cut-offs of 5,10 , and $15 \mathrm{~cm}$ would not be easily applicable to intracranial tumors, which grow in a confined space. However, necrosis was associated with decreased RFS on univariate analysis in our series. Furthermore, incorporating necrosis into ST-G, i.e., classifying tumors based on mitotic rate and necrosis, seems to improve the outcome models when compared to mitotic rate alone. In their recent work from 2018, Macagno et al. sought to validate an updated version of the Marseille Grading Scheme which segregated 132 tumors into three groups based on mitotic activity ( $\geq 5$ mitotic figures/10 HPF) and necrosis $[4,15]$. On univariate analysis, they found that extent of surgery, radiotherapy/chemotherapy, and mitotic rate were significant in predicting RFS, while radiation, mitotic rate, and necrosis were significant for disease-specific survival [15]. Extent of surgery and mitotic rate remained significant prognostic factors for RFS with multivariate analysis, while necrosis and radiotherapy were significant for disease-specific survival [15]. Our data parallel these findings, confirming that a grading scheme incorporating mitotic rate and necrosis best stratifies SFT/HPC, and the combination of high mitotic activity and necrosis portends poor prognosis for this entity. If these findings are corroborated by additional studies, this grading scheme could be utilized to guide treatment decisions regarding adjuvant therapy (e.g., radiotherapy) after surgery. Of note is the fact that, once necrosis is added to the ST-G model, the RFS curve of SFT does not appear to be significantly different from that of malignant SFT without necrosis, which, however, remains distinctly separate and inferior. Concern could be raised about applying the term malignant SFT as per the 2013 WHO classification for soft-tissue tumors to these tumors. Larger studies and longer follow-up are needed to answer this question.

In 2014, Barthelmess et al. examined a series of nonmeningeal SFT/HPC and showed that tumors harboring $N A B 2$ exon 4-STAT6 exon 2/3 fusions had lower recurrence rates than those with $N A B 2$ exon 6-STAT6 exon $16 / 17$ or other fusions [3]. Similarly, our subsequent work with meningeal-based lesions suggested a trend toward more aggressive behavior in tumors lacking NAB2 exon 4-STAT6 exon 3. Even though additional studies of dural- and nondural-based solitary fibrous tumors have shown consistent association between mitotic rate and fusion variant, with tumors harboring NAB2 exon 4-STAT6 exon 2/3 fusions consistently exhibiting lower mitotic rates than those with NAB2 exon 6-STAT6 exon $16 / 17$, no further relationship between fusion variant and disease-free survival has been reported $[1,3,6,12,18,21]$. Our current study that focused specifically on a large cohort of meningeal-based tumors from multiple large tertiary care centers provides further support that fusion status fails to associate with RFS or overall survival.

Interestingly, several studies, including our earlier work, have suggested that fusion type appears to be associated with phenotype. The NAB2 exon 4-STAT6 exon 2-3 variant seems to be most often found in tumors resembling the 
conventional 'solitary fibrous tumor,' while tumors with NAB2 exon 6-STAT6 exon 16-17 exhibit morphologic features consistent with what was previously considered 'hemangiopericytoma' $[1,3,11]$. Our current findings are concordant with these prior reports and suggest that tumor phenotype is related to fusion variant.

The sensitivity of STAT6 for the diagnosis of solitary fibrous tumors has been reported to range from 86 to $98 \%$ in large series at both CNS and soft-tissue sites [7, 9, 13, 17]. We identified only a single case of SFT/HPC which harbored an NAB2 exon 6-STAT6 exon 16 fusion but lacked nuclear STAT6 immunoreactivity. The tissue blocks from this patient were from 1997 and 1998, and it is possible that the age of the blocks precluded accurate immunohistochemical analysis. We also considered the possibility that the epitope recognized by our antibody may be lost in this specific fusion. However, the fusion in this case is the most frequently recognized, and, consequently, we would not expect loss of STAT6 staining based on fusion type. Regardless, the absence of STAT6 immunostaining in a tumor with morphologic features consistent with SFT/HPC should lead to the consideration of molecular testing to assess for the presence of NAB2-STAT6 fusion.

In conclusion, meningeal SFT/HPC is an aggressive tumor with propensity for high rates of recurrence and metastasis, which sometimes occur decades after the initial diagnosis. A grading scheme incorporating mitotic rate and necrosis seems to stratify this family of tumors most accurately. Although fusion type does not appear to relate to outcome in dural-based lesions, it does seem to be associated with tumor phenotype.

Acknowledgements We would like to acknowledge the Mayo Pathology Core for the nucleic acid extraction process.

\section{Funding Mayo Clinic.}

\section{Compliance with ethical standards}

Conflict of interest Dr. Sandro Santagata has the following disclosure: RareCyte, Inc. Dr. Brian M. Alexander has the following disclosure: Foundation Medicine, Inc. Dr. Priscilla K. Brastianos has the following disclosures: Honorarium from Genetech, Consultant for Merck, Lilly, and Angiochem and Research support from Merck.

Open Access This article is distributed under the terms of the Creative Commons Attribution 4.0 International License (http://creativeco mmons.org/licenses/by/4.0/), which permits unrestricted use, distribution, and reproduction in any medium, provided you give appropriate credit to the original author(s) and the source, provide a link to the Creative Commons license, and indicate if changes were made.

\section{References}

1. Akaike K, Kurisaki-Arakawa A, Hara K, Suehara Y, Takagi T, Mitani K et al (2015) Distinct clinicopathological features of NAB2-STAT6 fusion gene variants in solitary fibrous tumor with emphasis on the acquisition of highly malignant potential. Hum Pathol 46:347-356

2. Apra C, Mokhtari K, Cornu P, Peyre M, Kalamarides M (2018) Intracranial solitary fibrous tumors/hemangiopericytomas: first report of malignant progression. J Neurosurg 128:1719-1724

3. Barthelmess S, Geddert H, Boltze C, Moskalev EA, Bieg M, Sirbu $\mathrm{H}$ et al (2014) Solitary fibrous tumors/hemangiopericytomas with different variants of the NAB2-STAT6 gene fusion are characterized by specific histomorphology and distinct clinicopathological features. Am J Pathol 184:1209-1218

4. Bouvier C, Metellus P, de Paula AM, Vasiljevic A, Jouvet A, Guyotat J et al (2012) Solitary fibrous tumors and hemangiopericytomas of the meninges: overlapping pathological features and common prognostic factors suggest the same spectrum of tumors. Brain Pathol 22:511-521

5. Chmielecki J, Crago AM, Rosenberg M, O'Connor R, Walker SR, Ambrogio L et al (2013) Whole-exome sequencing identifies a recurrent NAB2-STAT6 fusion in solitary fibrous tumors. Nat Genet 45:131-132

6. Chuang IC, Liao KC, Huang HY, Kao YC, Li CF, Huang SC et al (2016) NAB2-STAT6 gene fusion and STAT6 immunoexpression in extrathoracic solitary fibrous tumors: the association between fusion variants and locations. Pathol Int 66:288-296

7. Demicco EG, Harms PW, Patel RM, Smith SC, Ingram D, Torres $\mathrm{K}$ et al (2015) Extensive survey of STAT6 expression in a large series of mesenchymal tumors. Am J Clin Pathol 143:672-682

8. Demicco EG, Wagner MJ, Maki RG, Gupta V, Iofin I, Lazar AJ et al (2017) Risk assessment in solitary fibrous tumors: validation and refinement of a risk stratification model. Mod Pathol 30:1433-1442

9. Doyle LA, Vivero M, Fletcher CD, Mertens F, Hornick JL (2014) Nuclear expression of STAT6 distinguishes solitary fibrous tumor from histologic mimics. Mod Pathol 27:390-395

10. Fletcher CDM, Hogendoorn PCW, Mertens F (2013) World Health Organization classification of tumours. In: Fletcher CDM, Bridge JA, Hogendoorn PCW, Mertens F (eds) WHO classification of tumours of soft tissue and bone, vol 1, 4th edn. International Agency for Research on Cancer, Lyon, pp 80-82

11. Fritchie KJ, Jin L, Rubin BP, Burger PC, Jenkins SM, Barthelmess $S$ et al (2016) NAB2-STAT6 gene fusion in meningeal hemangiopericytoma and solitary fibrous tumor. J Neuropathol Exp Neurol $75: 263-271$

12. Huang SC, Li CF, Kao YC, Chuang IC, Tai HC, Tsai JW et al (2016) The clinicopathological significance of NAB2-STAT6 gene fusions in 52 cases of intrathoracic solitary fibrous tumors. Cancer Med 5:159-168

13. Koelsche C, Schweizer L, Renner M, Warth A, Jones DT, Sahm F et al (2014) Nuclear relocation of STAT6 reliably predicts NAB2/ STAT6 fusion for the diagnosis of solitary fibrous tumour. Histopathology 65:613-622

14. Louis DN, Ohgaki H, Wiestler OD, Cavenee WK, Ellison DW, Figarella-Branger D et al (2016) World Health Organization classification of tumours. In: Louis DN, Ohgaki H, Wiestler OD, Cavenee WK (eds) WHO classification of tumours of the central nervous system, vol 1, 4th edn. International Agency for Research on Cancer, Lyon, pp 249-254

15. Macagno N, Vogels R, Appay R, Colin C, Mokhtari K, French CNSSFTHPCC et al (2018) Grading of meningeal solitary fibrous tumors/hemangiopericytomas: analysis of the prognostic value of 
the Marseille Grading System in a cohort of 132 patients. Brain Pathol. https://doi.org/10.1111/bpa.12613

16. Robinson DR, Wu YM, Kalyana-Sundaram S, Cao X, Lonigro RJ, Sung YS et al (2013) Identification of recurrent NAB2-STAT6 gene fusions in solitary fibrous tumor by integrative sequencing. Nat Genet 45:180-185

17. Schweizer L, Koelsche C, Sahm F, Piro RM, Capper D, Reuss DE et al (2013) Meningeal hemangiopericytoma and solitary fibrous tumors carry the NAB2-STAT6 fusion and can be diagnosed by nuclear expression of STAT6 protein. Acta Neuropathol 125:651-658

18. Tai HC, Chuang IC, Chen TC, Li CF, Huang SC, Kao YC et al (2015) NAB2-STAT6 fusion types account for clinicopathological variations in solitary fibrous tumors. Mod Pathol 28:1324-1335
19. Team RC (2017) R: a language and environment for statistical computing. R Foundation for Statistical Computing. https:// www.R-project.org/. Accessed 1 June 2018

20. Wang X, Bledsoe KL, Graham RP, Asmann YW, Viswanatha DS, Lewis JE et al (2014) Recurrent PAX3-MAML3 fusion in biphenotypic sinonasal sarcoma. Nat Genet 46:666-668

21. Yuzawa S, Nishihara H, Wang L, Tsuda M, Kimura T, Tanino M et al (2016) Analysis of NAB2-STAT6 gene fusion in 17 cases of meningeal solitary fibrous tumor/hemangiopericytoma: review of the literature. Am J Surg Pathol 40:1031-1040

\section{Affiliations}

Karen Fritchie ${ }^{1}\left(\right.$ Kassandra Jensch $^{2} \cdot$ Evgeny A. Moskalev $^{2} \cdot$ Alissa Caron $^{1} \cdot$ Sarah Jenkins $^{3} \cdot$ Michael Link $^{4}$. Paul D. Brown ${ }^{5}$. Fausto J. Rodriguez ${ }^{6}$. Andrew Guajardo ${ }^{6}$. Daniel Brat ${ }^{7}$. José E. Velázquez Vega ${ }^{8}$. Arie Perry ${ }^{9}$. Ashley $\mathrm{Wu}^{9}$. David R. Raleigh ${ }^{10,11}$ - Sandro Santagata ${ }^{12}$ - David N. Louis ${ }^{13}$. Priscilla K. Brastianos ${ }^{14}$. Alexander Kaplan ${ }^{15} \cdot$ Brian M. Alexander $^{16} \cdot$ Sabrina Rossi $^{17} \cdot$ Fabio Ferrarese $^{18} \cdot$ Florian Haller $^{2} \cdot$ Caterina Giannini $^{1}$

1 Anatomic Pathology, Department of Laboratory Medicine and Pathology, Mayo Clinic, 200 First Street, SW, Rochester, MN 55905, USA

2 Institute of Pathology, University Hospital Erlangen, Erlangen, Germany

3 Department of Health Sciences Research, Mayo Clinic, Rochester, MN, USA

4 Department of Neurosurgery, Mayo Clinic, Rochester, MN, USA

5 Department of Radiation Oncology, Mayo Clinic, Rochester, MN, USA

6 Department of Pathology, Johns Hopkins, Baltimore, MD, USA

7 Department of Pathology, Northwestern University Feinberg School of Medicine, Chicago, IL, USA

8 Department of Pathology, Children's Healthcare of Atlanta, Atlanta, GA, USA

9 Department of Pathology, University of California, San Francisco, CA, USA
10

Department of Radiation Oncology, University of California, San Francisco, CA, USA

11 Department of Neurological Surgery, University of California, San Francisco, CA, USA

12 Department of Pathology, Brigham and Women's Hospital, Boston, MA, USA

13 Department of Pathology, Massachusetts General Hospital and Harvard Medical School, Boston, MA, USA

14 Department of Hematology/Oncology, Massachusetts General Hospital and Harvard Medical School, Boston, MA, USA

15 Department of Neuro-oncology, Massachusetts General Hospital and Harvard Medical School, Boston, MA, USA

16 Department of Radiation Oncology, Dana-Farber Cancer Institute, Boston, MA, USA

17 Department of Pathology and Molecular Genetics, Ospedale Ca'Foncello, Treviso, Italy

18 Department of Radiation Oncology, Ospedale Ca'Foncello, Treviso, Italy 